\title{
Review: antidepressants are not effective for cocaine dependence
}

Lima MS, Reisser AA, Soares BG, et al. Antidepressants for cocaine dependence. Cochrane Database Syst Rev 2001;(4):CD002950 (latest version 27 Aug 2001).

\section{QUESTION: In patients with cocaine dependence, is antidepressant drug treatment more effective than placebo for reducing cocaine use?}

\section{Data sources}

Studies were identified by searching the Cochrane Controlled Trials Register, Medline (1966-2000), EMBASE/ Excerpta Medica (1980-2000), LILACS (1982-2000), PsycLIT (1974-2000), Biological Abstracts (19822000), and the trials' registers of 3 Cochrane review groups; by reviewing book chapters on the treatment of cocaine dependence; and by contacting pharmaceutical companies and experts in the field.

\section{Study selection}

Studies were selected if they were randomised controlled trials (RCTs) that compared antidepressant drugs with placebo, other medication, or psychosocial interventions in patients with cocaine dependence (including those with an additional diagnosis of opioid dependence or in methadone maintenance schemes).

\section{Data extraction}

2 reviewers independently extracted data on setting; patient characteristics; intervention, including type of antidepressant and dose; study duration; study quality, including concealment and blinding; and outcomes, including cocaine metabolites in urine sample, dropouts, and side effects.

\section{Main results}

20 RCTs (1304 patients, age range 20-60 y) were included. 14 trials studied desipramine, 2 studied fluoxetine, and 1 RCT each studied ritanserin, gepirone, bupropion, and imipramine. Study duration ranged from 40 days to 6 months. Antidepressants did not differ from placebo for positive urine samples for cocaine metabolites, dropouts, or side effects (table).

\section{Conclusion}

In patients with cocaine dependence, antidepressants are not effective for reducing cocaine use.

Antidepressants $v$ placebo for cocaine dependence at 40 days to 6 months*

Sources of funding: Institute of Psychiatry, London, UK; World Health Organization Universidade Federal de Pelotas, Brazil.

For correspondence: Dr M S Lima,

Universidade Federal de Pelotas, Rio Grande do Sul, Brazil.

mslima@nutecnet.com.br

\begin{tabular}{lccll} 
Outcomes for cocaine dependence & No of trials & Weighted event rates & RRR (95\% CI) & NNT \\
\hline Cocaine metabolites in urine sample & 7 & $53 \% v 56 \%$ & $6 \%(-30$ to 32$)$ & Not significant \\
\hline Dropouts & 8 & $45 \% v 49 \%$ & $12 \%(-19$ to 35$)$ & Not significant \\
\hline $\begin{array}{l}\text { Outcomes for opioid/ methadone maintenance } \\
\text { Cocaine metabolites in urine sample }\end{array}$ & 2 & $59 \% v 32 \%$ & $65 \%(-2$ to 179$)$ & Not significant \\
\hline$\geqslant 1$ side effect & 2 & & RRR (CI) & NNT \\
\hline Dropouts & 1 & $48 \% v 85 \%$ & $56 \%(-193$ to 93$)$ & Not significant \\
\hline
\end{tabular}

*Abbreviations defined in glossary; RRR, RRI, NNT, NNH, and $\mathrm{Cl}$ calculated from data in article using a random effects model.

†Event rates not weighted.

\section{COMMENTARY}

Cocaine abuse remains a substantial public health problem in the US and throughout the world. Numerous studies in recent years have tested a long list of putative pharmacotherapies for cocaine abuse. Finding effective pharmacological agents has been a major focus of research funded by the US National Institute on Drug Abuse (NIDA). 2 recent reviews from the Cochrane Collaboration by Lima et al and Soares et al focus on the most widely tested classes of medications: antidepressants and dopamine agonists.

Hopes were raised in the 1980s and early 1990s that antidepressants might be useful in reducing cocaine use, craving, or relapse. Clinical observations had shown that patients had substantial depressive symptoms during cocaine withdrawal. Preclinical research also showed that antidepressants modulate the same monoamine neurotransmitters that are affected by cocaine use. Early studies on antidepressants, particularly those involving the use of desipramine and fluoxetine, suggested that these agents might reduce cocaine use. However, later trials with these and with other antidepressants, such as bupropion, have been largely negative and have failed to fulfill the promise shown in early studies.

The review by Lima $e t$ al reaches a definite conclusion: antidepressants are ineffective for reducing cocaine use. This clearly negative finding was reached even though the authors did not include among the 20 RCTs in their analysis 2 other carefully done negative trials-those of Grabowski $e t$ al. ${ }^{1}$

Although the results of studies with antidepressants in cocaine treatment appear to be overwhelmingly negative, one important question remains which was not addressed in the review-whether the presence or absence of major depressive disorder might influence treatment outcomes. It is reasonable to consider the possibility that antidepressants may have clinical value in depressed cocaine abusers even if they are ineffective for cocaine abuse in general. This question could be answered by future studies in which cocaine dependent patients are stratified before randomisation according to the presence or absence of major depressive disorder.

A second major direction taken in the search for medications to treat cocaine abuse involves the use of dopamine agonists, as reviewed by Soares $e t$ al in their analysis of 12 RCTs. Early optimism about dopamine agonists as effective pharmacotherapies for cocaine dependence was supported by the knowledge that cocaine produces euphoria and arousal because of acute inhibition of dopamine reuptake, although continued use leads to chronic dopamine depletion. Efforts were made to find dopamine agonists that might diminish cocaine craving and cocaine use. Early studies focused on bromocriptine and amantadine, and included some positive results, but more recent trials have been notably negative. Continued on next page 


\section{Review: dopamine agonists are not effective for cocaine dependence}

Soares BG, Lima MS, Reisser AA, et al. Dopamine agonists for cocaine dependence. Cochrane Database Syst Rev 2001;(4);CD003352 (latest version 27 Aug 2001).

\section{QUESTION: In patients with cocaine dependence, are dopamine agonists effective for reducing cocaine use?}

\section{Data sources}

Studies were identified by searching the Cochrane Controlled Trials Register, Medline (1966-2000), EMBASE/ Excerpta Medica (1980-2000), LILACS (1982-2000), PsycLIT (1974-2000), Biological Abstracts (19822000), and the trial registers of 3 Cochrane Review Groups; contacting authors and pharmaceutical manufacturers; and reviewing book chapters on the treatment of cocaine dependence.

\section{Study selection}

Studies were selected if they were randomised controlled trials (RCTs) that compared dopamine agonists with placebo, other medications, or psychosocial interventions in patients with cocaine dependence (including those with an additional diagnosis of opioid dependence or those in methadone maintenance schemes).

\section{Data extraction}

2 reviewers independently extracted data on patient characteristics; study duration; setting; intervention, including dose and regimen; study quality; and outcomes, including positive urine sample for cocaine metabolites and treatment retention.

\section{Main results}

12 RCTs (587 patients) were included. Study duration ranged from 10 days to 12 weeks. 9 RCTs were done in outpatients in the community or in mental health centres, 1 RCT was done in day hospital patients, and 2 RCTs were done in inpatients. 1 small RCT $(n=14)$ showed a reduction in dropouts for amantadine relative to bromocriptine; no other statistically significant difference was seen for any other outcome (table).

\section{Conclusion}

In patients with cocaine dependence, dopamine agonists are not effective for reducing cocaine use.
Sources of funding:

Institute of Psychiatry London, UK; World Health Organisation; Universidade Federal de Pelotas, Brazil.

For correspondence: Dr B G Soares, Centro Cochrane do Brasile, Sao Paulo, Brazil. bgos@zaz.com.br

Dopamine agonists (DAs) for cocaine dependence at 10 days to 12 weeks*

\begin{tabular}{|c|c|c|c|c|}
\hline Outcomes for cocaine dependence & Comparisons (number of studies) & Weighted event rates & RRR (95\% CI) & NNT \\
\hline \multirow[t]{4}{*}{ Cocaine metabolites in urine sample } & DA $v$ plac $(2)$ & $39 \% \vee 63 \%$ & $49 \%(-114$ to 88$)$ & Not significant \\
\hline & Amant $v$ bromo (1) & $29 \%$ v 57\%† & $50 \%(-68$ to 87$)$ & Not significant \\
\hline & & & RRI (Cl) & NNH \\
\hline & Amant $v$ desi (1) & $78 \% \vee 75 \% \dagger$ & $4.3 \%(-26$ to 42$)$ & Not significant \\
\hline \multirow[t]{4}{*}{ Dropouts } & DA $v$ plac $(5)$ & $51 \%$ v $44 \%$ & $14 \%(-9$ to 43$)$ & Not significant \\
\hline & & & $\operatorname{RRR}(\mathrm{Cl})$ & NNT (Cl) \\
\hline & Amant $v$ desi (1) & $83 \%$ v $84 \% \dagger$ & $2.1 \%(-26$ to 27$)$ & Not significant \\
\hline & Amant $v$ bromo (1) & $29 \% v 86 \% \dagger$ & $67 \%(9.3$ to 91$)$ & $2(2$ to 30$)$ \\
\hline \multicolumn{2}{|c|}{ Outcomes for opioid/methadone maintenance } & & RRI (Cl) & NNH \\
\hline \multirow[t]{2}{*}{ Cocaine metabolites in urine sample } & Amant $v$ plac (3) & $80 \% \vee 78 \%$ & $2 \%(-13$ to 20$)$ & Not significant \\
\hline & Amant $v$ desi (2) & $97 \% \vee 61 \%$ & $118 \%$ (-63 to 1193$)$ & Not significant \\
\hline \multirow[t]{2}{*}{ Dropouts } & Amant $v$ plac (4) & $37 \%$ v $35 \%$ & $3 \%(-18$ to 29$)$ & Not significant \\
\hline & Amant $v$ desi (2) & $36 \% \vee 21 \%$ & $68 \%(-75$ to 1041$)$ & Not significant \\
\hline
\end{tabular}

*Amant=amantadine; bromo=bromocriptine; desi=desipramine; plac=placebo. Other abbreviations defined in glossary; RRR, RRI, NNT, NNH, and Cl calculated from data in article using a random effects model.

†Event rates not weighted.

\section{COMMENTARY-continued}

Both of these Cochrane reviews are affected by the difficulties inherent in pooling the various studies of antidepressants and dopamine agonists, respectively. Particularly daunting are the limitations imposed by the heterogeneity of the patients in the different studies and by the variations in research methodology and outcome measures.

These 2 reviews, summarising 32 RCTs, leave clinicians with the very clear message that, as of yet, no medication offers a ready answer to the problem of cocaine addiction, and that patients are therefore best treated with non-pharmacological methods. Psychosocial approaches include interventions ranging from cognitive psychotherapy to behaviour therapy, such as contingency management. Clinical trials that provide material incentives for abstinence from cocaine $^{2}$ have shown generally far more robust decreases in cocaine use than have medications. For now, cognitive and behavioural treatments remain the standard care and treatment of choice for cocaine dependence.

1 Grabowski J, Rhoades H, Elk R, et al. Fluoxetine is ineffective for treatment of cocaine dependence or concurrent opiate and cocaine dependence: two placebo-controlled double-blind trials. J Clin Psychopharmacol 1995;15:163-74.

2 Higgins ST, Wong CJ, Badger GJ, et al. Contingent reinforcement increases cocaine abstinence during outpatient treatment and 1 year of follow-up.J Consult Clin Psychol 2000;68:64-72. 